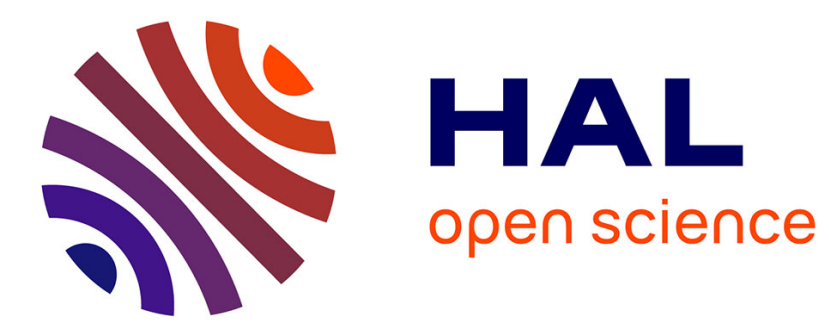

\title{
Travelling Ultrasonic Wave Enhances Keyclick Sensation
} David Gueorguiev, Anis Kaci, Michel Amberg, Frédéric Giraud, Betty

\author{
Lemaire-Semail
}

\section{To cite this version:}

David Gueorguiev, Anis Kaci, Michel Amberg, Frédéric Giraud, Betty Lemaire-Semail. Travelling Ultrasonic Wave Enhances Keyclick Sensation. EuroHaptics 2018 - 11th International Conference on Haptics: Science, Technology, and Applications, Jun 2018, Pise, Italy. hal-01905646

\section{HAL Id: hal-01905646 https://hal.science/hal-01905646}

Submitted on 26 Oct 2018

HAL is a multi-disciplinary open access archive for the deposit and dissemination of scientific research documents, whether they are published or not. The documents may come from teaching and research institutions in France or abroad, or from public or private research centers.
L'archive ouverte pluridisciplinaire HAL, est destinée au dépôt et à la diffusion de documents scientifiques de niveau recherche, publiés ou non, émanant des établissements d'enseignement et de recherche français ou étrangers, des laboratoires publics ou privés. 


\title{
Travelling Ultrasonic Wave Enhances Keyclick Sensation
}

\author{
David Gueorguiev ${ }^{1,3}$, Anis Kaci², Michel Amberg ${ }^{2}$, Frédéric Giraud ${ }^{2}$, and \\ Betty Lemaire-Semail ${ }^{2}$ \\ 1 Inria Lille Nord Europe, 59650 Villeneuve d'Asq, France. \\ 2 Univ. Lille, Centrale Lille, Arts et Métiers Paris Tech, HEI, EA 2697 - L2EP - \\ F-59000 Lille, France anis.kaci@etudiant.univ-lille1.fr, \\ Michel.Amberg@univ-lille1.fr, frederic.giraud@polytech-lille.fr \\ 3 Max-Planck Institute for Intelligent Systems, 70569 Stuttgart, Germany \\ dgueorguiev@is.mpg.de
}

\begin{abstract}
A realistic keyclick sensation is a serious challenge for haptic feedback since vibrotactile rendering faces the limitation of the absence of contact force as experienced on physical buttons. It has been shown that creating a keyclick sensation is possible with stepwise ultrasonic friction modulation. However, the intensity of the sensation is limited by the impedance of the fingertip and by the absence of a lateral force component external to the finger. In our study, we compare this technique to rendering with an ultrasonic travelling wave, which exerts a lateral force on the fingertip. For both techniques, participants were asked to report the detection (or not) of a keyclick during a forced choice one interval procedure. In experiment 1, participants could press the surface as many time as they wanted for a given trial. In experiment 2, they were constrained to press only once. The results show a lower perceptual threshold for travelling waves. Moreover, participants pressed less times per trial and exerted smaller normal force on the surface. The subjective quality of the sensation was found similar for both techniques. In general, haptic feedback based on travelling ultrasonic waves is promising for applications without lateral motion of the finger.
\end{abstract}

Keywords: haptic display; tactile perception; ultrasonic vibration; travelling wave; keyclick; button click

\section{Introduction}

Haptic interaction is essential to our capacity to use portable interactive screens without physical keyboard [1]. In the last decades, tactile displays have greatly improved with the development of naive on-screen physics that are intuitive to the user [20] and rapid progress in the ability to deliver force-based tactile feedback $[15,11]$. However, it is still difficult to type a large text on the keyboard of a tablet. The difficulty of this task partly comes from the ergonomics of the screen but also from the lack of tactile feedback that current tactile displays provide to us while typing. At best, they deliver timely vibrations that replace the 
a)

Ultrasonic motor

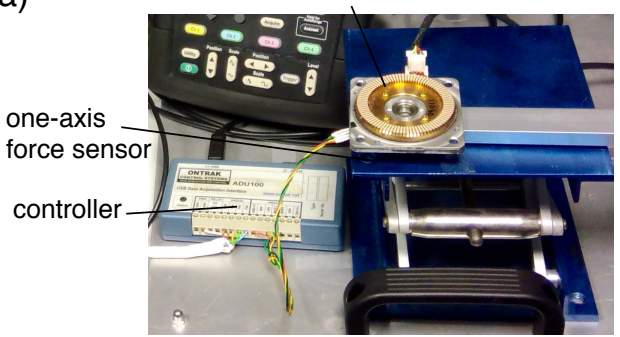

b)

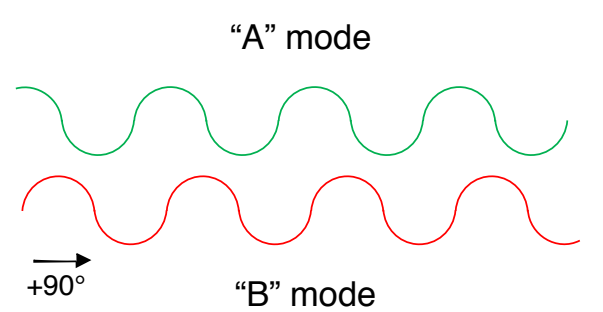

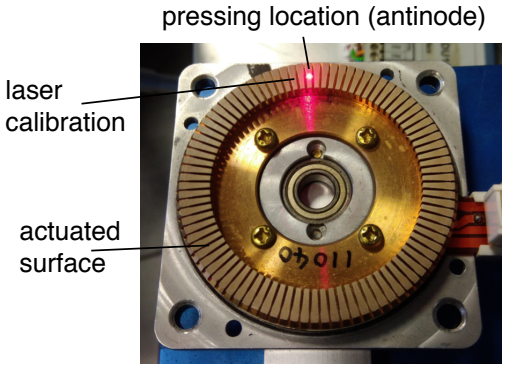

c)

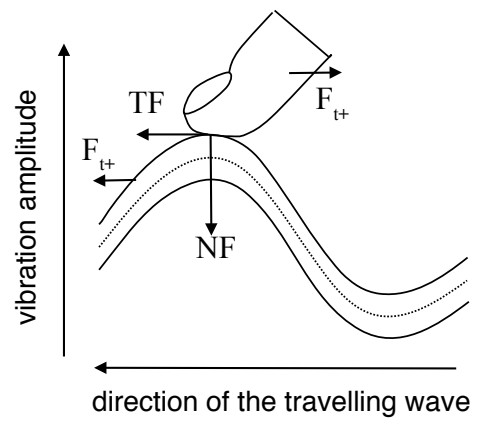

Fig. 1: a) The apparatus used for the generation of the keyclick. The actuation was performed on an ultrasonic motor. b) The ultrasonic travelling wave was generated by the superposition of two stationary waves, which are shifted by either $-90^{\circ}, 0^{\circ}$ or $+90^{\circ}$. c) The travelling wave generates an additional tangential force $F_{t+}$ on the fingertip compared to the stationary ultrasonic vibration.

contact mechanics occurring during the push of a classical keyboard button. The absent or impaired sensation compared to an old-fashioned physical keyboard is an important shortcoming of the current smartphones and tablets and one of the major challenges ahead of the realistic rendering of haptic feedback. In addition to the poor user experience, unnatural feedback also impairs the user's typing performance on the device. Several vibration-based technologies for creating a button-click have been suggested. $250 \mathrm{~Hz}$ vibration with up and down ramps have proven effective to improve finger-based text entry [7]. It was also suggested that a higher fidelity of the vibrotactile signal hence a more pleasant click sensation could be achieved by using piezo actuators instead of conventional vibrating motors [8]. A recent study showed that, in addition to the improved performance, the feeling of an haptic click can be recreated by using three repeated cycles of a $250 \mathrm{~Hz}$ sinusoidal signal generated by piezo actuators $[2,10]$.

However, vibrotactile feedback is inherently different to the force feedback generated by the keys of physical keyboards, which apply contact forces to the fingertip [18]. It has been suggested that a button click sensation can be achieved by modulating the friction between a finger and an ultrasonically vibrating surface at the moment of contact in order to recreate the rapid change in force 
induced by the buckling of a mechanical push button [14]. Indeed, the human perception of transient frictional cues is particularly accurate at the onset of the tactile interaction with a surface and [5] and event-base feedback is known to create a more realistic haptic sensation [9] compared to position-based feedback. Therefore, frictional modulations driven by the user's behavior are very salient for the sense of touch and small differences in their timing or sharpness can have a large perceptual impact [4].

\section{Earlier work}

A recent study has built on these principles to show that a realistic keyclick sensation can be generated by an event-based stepwise modulations of the ultrasonic vibration [12]. Two types of stimulation were tried: a step of falling friction and a step of rising friction occurring at a predefined normal force level. Users detected more easily the falling friction than the rising friction and reported to feel it as a convincing keyclick sensation. The proposed method relies on sudden changes in the impedance of the finger [13], which are provoked by the influence of the ultrasonic lubrication $[19,16]$ on the compression of the fingerpad. Lateral motion of the finger is generally necessary to the occurrence of friction modulation by ultrasonic lubrication. In its absence, the subtle mechanics generating the keyclick sensation require a high amplitude of ultrasonic vibration to be perceivable and the threshold for perceiving the clickbutton is several times higher than the threshold for perceiving frictional steps during dynamic tactile exploration [6]. The quality of the sensation was also found to be strongly influenced by the natural impedance of the fingertip with an impaired sensation for extreme impedances.

It is also possible to enhance the modulation of friction hence the tactile sensation generated by ultrasonic actuation on the fingertip through the implementation of an ultrasonic travelling wave instead of a stationary wave. When vibrating, the particles of a surface on which a travelling wave is propagating undergo an elliptic motion. For specific operating conditions, they can help to propel the finger if they move in the same direction as the finger pulp when they come in contact with it. This effect additionally decreases the equivalent friction coefficient compared with a pure stationary wave [3]. Conversely, a travelling wave decreases the the friction reduction when the direction is reversed.

Progressive ultrasonic waves can be wisely used to generate a keyclick sensation during pressing on an actuated surface. Our method consists in a stepwise switching on of a travelling ultrasonic wave at a predefined normal force threshold and a reversal of its direction when a second predefined normal force threshold is reached. This method exploits the frictional force exerted by the ultrasonic travelling wave to deliver a click sensation to the user during the pressing of the surface. This study aims to compare both methods for generating a keyclick sensation in terms of psychophysical threshold, quality of the sensation and pressing behavior of the participants. In a first experiment, we let participants free to explore the sensation by not restricting the number of pressings by trial in order 
a)

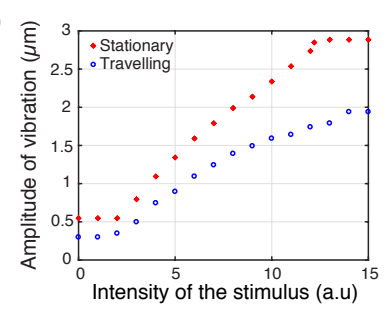

b)

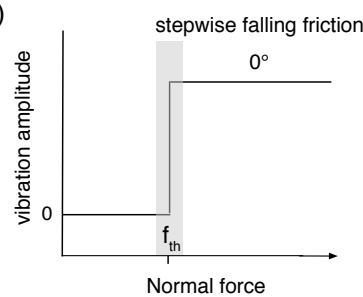

c)

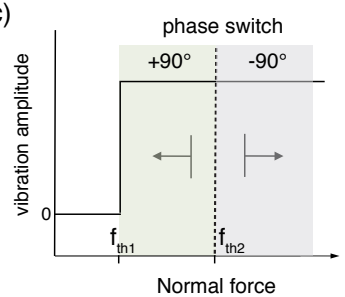

Fig. 2: a) The correspondance between the arbitrary intensity values and the amplitude of ultrasonic vibration. b) The keyclick actuated by the stationary wave was generated by a stepwise switch on of the ultrasonic vibration at a predefined threshold value of the normal force c) For the travelling wave, the stepwise increase in amplitude at $f_{t h 1}$ is followed by a second normal force threshold $f_{t h 2}$ at which the direction of the ultrasonic wave is reversed.

to see if they would choose different exploratory strategies for the two methods. In a second experiment, we increased the comparability between the methods by estimating the perceptual threshold when participants were constrained to press only once by trial on the surface.

\section{Materials and Methods}

\subsection{Participants}

Data were collected from 20 healthy volunteers aged between 22 and 62 (5 females). 10 participated in experiment 1 and 10 additional participants were recruited for experiment 2. Participants were wearing noise-cancelling headphones in order to prevent potential interference from auditory cues. All participants gave written informed consent. The investigation conformed to the principles of the Declaration of Helsinki and experiments were performed in accordance with relevant guidelines and regulations.

\section{$3.2 \quad$ Experimental set-up}

For this experiment, the stator of a USR60 ultrasonic motor (Shinsei Corporation, Japan) was used (Fig. 1a). It is constituted by a bronze disk on which a ring of 16 piezoelectric actuators is glued. Half of these actuators are arranged so that they can excite the $9^{t h}$ bending mode denoted by $\cos$ hereafter, which is characterized by a resonance frequency of $40 \mathrm{kHz}$, and a wavelength $\lambda=21 \mathrm{~mm}$. The other actuators are arranged with a spatial shift of $\lambda / 4$ on the ring, thus exciting a doublet of the $\cos$ mode, which is denoted $\sin$. The excitation of modes cos and $\sin$ in quadrature, i.e. with a temporal phase shift of $\pi / 2$ (Fig. 1b) produces a travelling wave (Fig. 1c). Changing the sign of the phase shift will invert the direction of the wave; cancelling the phase shift produces a stationary wave. A 
a)

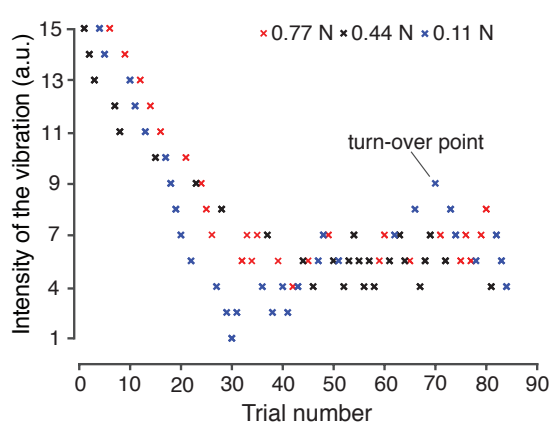

b)

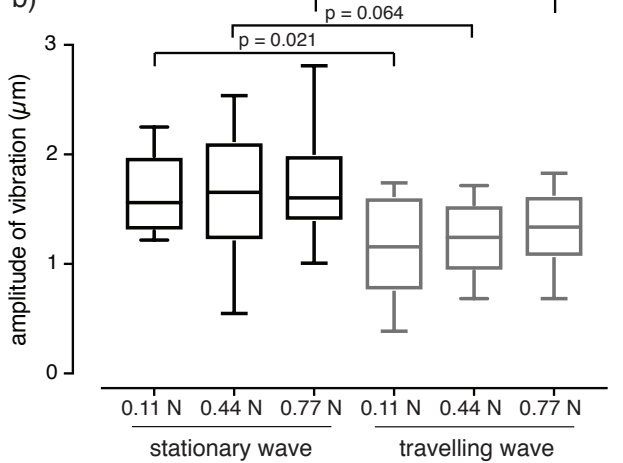

Fig. 3: a) Typical example of three interlaced psychophysical staircases, which target the $50 \%$ perceptual threshold by a one-up one-down paradigm. At each trial, the implemented staircase is chosen at random with a probability of $1 / 3$. b) In experiment 1 (multiple clicks), the $50 \%$ perceptual threshold was computed for both actuation techniques. The error bars, the whisker boxes and the horizontal bars show respectively the min. and max. values, their interquartile range and the median value.

laser doppler vibrometer (OFV 505, Polytec, Germany) was used to localize the antinodes of the stationary wave, and was marked as a target for the pressing. The normal force during pressing on the actuated surface was measured by a one-axis force sensor, located below the pressing area, with a resolution of 0.01 $\mathrm{N}$. The acquisition of the force values was performed by a in-house acquisition board at a $42 \mathrm{~Hz}$ sampling rate. In addition, the laser vibrometer was used to compute the values in $\mu \mathrm{m}$ corresponding to the arbitrary units (from 1 to 15 ) controlling the intensity of the ultrasonic vibration (Fig. 2a).

The keyclick rendering when using a stationary wave was performed as in [12] by a step increase of the amplitude of vibration when a predefined threshold of the normal force was reached (Fig. 2b). For the travelling wave method, two normal force thresholds $f_{t h 1}$ and $f_{t h 2}$ were predefined (Fig. 2c). At $f_{t h 1}$, the travelling wave was switched on by a step increase in the amplitude of vibration. Above the normal force threshold $f_{t h 2}$, which was set $0.33 \mathrm{~N}$ higher than $f_{t h 1}$, the direction of the travelling wave was reversed in order to enhance the tactile feedback on the fingertip.

\subsection{Experimental procedure}

In both experiments, participants were asked to press on the actuated surface with their index finger. The target location was chosen on a vibration antinode and labelled with a color marker in order to ensure that participants experienced consistent ultrasonic vibration across trials. The estimation of the psychophysical threshold was performed with a one-up one-down staircase procedure, which 
targeted the intensity at which a keyclick sensation was felt $50 \%$ of the time, a procedure also referred as Bekesy tracking method [17]. At each trial, after pressing on the actuated surface, the participants had to report if they felt a keyclick sensation or not. We chose a non-forced choice procedure despite its proneness for criterion bias for two reasons: $1 /$ we wanted the participants to focus on the keyclick sensation rather than on the perception of the variation of the finger-surface friction. 2/ for a given participant, the psychophysical estimation for the progressive and stationary wave are likely to be identically influenced by its criterion bias. Thus, the influence of the criterion on the comparison is lower than if the aim of the study was to estimate the absolute psychophysical threshold. Before each block, participants were allowed to familiarize themselves with the haptic feedback by testing several clicks at maximum intensity.

To avoid potential biases due to prediction of the next stimulation intensity by participants, three staircases with different force thresholds were interlaced $(0.11,0.44$ and $0.77 \mathrm{~N})$. For each trial, the probability to be presented with a trial from a given staircase was $1 / 3$ (Fig. 3a). The experiment ended when 5 turnovers or 30 trials were achieved for all three staircases. For each staircase, the perceptual threshold was then computed as the mean of the last 3 turnovers. One participant in experiment 2 made less than three turnovers in one of the conditions and we had to discard its data. The experiment was performed two times: one with the stationary wave method and another time with the progressive wave method. The order of the two conditions was pseudo-randomized across participants to avoid learning curve effects.

\section{Results}

\subsection{Multiple clicks}

For all tested actuation criteria $(0.11 \mathrm{~N}, 0.44 \mathrm{~N}$ and $0.77 \mathrm{~N})$, we computed the $50 \%$ detection thresholds for both methods to generate a keyclick sensation (Fig 3.b). For the stationary ultrasonic vibration, the median value of the individual thresholds for $0.11 \mathrm{~N}, 0.44 \mathrm{~N}$ and $0.77 \mathrm{~N}$ were respectively $1.56 \mu \mathrm{m}(\mathrm{IQR}=$ $1.98-1.30), 1.65 \mu \mathrm{m}(\mathrm{IQR}=2.11-1.21)$ and $1.60 \mu \mathrm{m}(\mathrm{IQR}=2.00-1.39)$. For the travelling ultrasonic vibration, the median values of the individual thresholds for $0.11 \mathrm{~N}, 0.44 \mathrm{~N}$ and $0.77 \mathrm{~N}$ were respectively $1.16 \mu \mathrm{m}(\mathrm{IQR}=1.61-0.76), 1.24 \mu \mathrm{m}$ $(\mathrm{IQR}=1.54-0.94)$ and $1.34 \mu \mathrm{m}(\mathrm{IQR}=1.62-1.06)$. The results showed a significantly lower threshold for the rendering technique based on travelling ultrasonic vibration (Friedman test: $\chi 2=16.09, \mathrm{p}=0.0066$ ). A further post-hoc analysis with Wilcoxon matched-pairs signed rank test showed that the difference between the two techniques was statistically significant for the $0.11 \mathrm{~N}$ and $0.77 \mathrm{~N}$ thresholds (respectively $\mathrm{n}=10, \mathrm{~W}=-44, \mathrm{p}=0.0215$ and $\mathrm{n}=10, \mathrm{~W}=-49, \mathrm{p}$ $=0.001)$ while a trend was observed for the $0.44 \mathrm{~N}$ threshold $(\mathrm{n}=10, \mathrm{~W}=-37$, $\mathrm{p}=0.064)$. Overall, rendering with travelling ultrasonic vibration decreased the detection threshold by around $25-30 \%$ compared to actuation with a stationary ultrasonic wave. 
In the first experiment, participants were allowed to press as many times as they wanted on the actuated surface for a given trial. For each participant, the average number of presses across the experiment did not differ significantly between the two actuation methods and the three possible force thresholds (nonparametric Friedman test: $\chi 2=7.77, \mathrm{p}=0.1693$ ) (Fig. 4a). However, large numbers of consecutive surface presses (clicks) were mostly found close to the psychophysical threshold. Thus, we computed the average number of clicks across participants for each trial number and we compared both techniques for the first 52 trials (Fig. 4b), which was the number of trials available for all participants. The results showed a significantly higher average number of clicks for the actuation by a stationary wave (Wilcoxon matched-pairs signed rank statistical: $\mathrm{N}=52, \mathrm{~W}=712, \mathrm{p}=0.0006)$. Similarly, we computed the peak force during pressing for the first 52 trials and compared it for both techniques (Fig. 4c). The peak force during pressing was found significantly higher for the actuation with a stationary wave (Wilcoxon matched-pairs signed rank statistical: $\mathrm{N}=$ $52, \mathrm{~W}=1022, \mathrm{p}<0.0001)$. Overall, these results show that, in the case of the stationary wave, the participants tended to press more times and harder when the actuation intensity was close to their psychophysical threshold.

\subsection{One click}

In the second experiment, participants were instructed to press only once per trial on the actuated surface. For the stationary ultrasonic vibration, the median value of the individual thresholds for $0.11 \mathrm{~N}, 0.44 \mathrm{~N}$ and $0.77 \mathrm{~N}$ were found to be respectively $1.86 \mu \mathrm{m}(\mathrm{IQR}=2.85-1.04), 1.88 \mu \mathrm{m}(\mathrm{IQR}=2.73-1.26)$ and $2.27 \mu \mathrm{m}(\mathrm{IQR}=2.85-1.75)$. For the progressive ultrasonic vibration, the medians of the individual thresholds for $0.11 \mathrm{~N}, 0.44 \mathrm{~N}$ and $0.77 \mathrm{~N}$ were respectively $1.49 \mu \mathrm{m}$ $(\mathrm{IQR}=1.75-0.51), 1.54 \mu \mathrm{m}(\mathrm{IQR}=1.73-0.37)$ and $1.63 \mu \mathrm{m}(\mathrm{IQR}=1.82-0.62)$ (Fig. 4d). The results showed a significantly lower threshold for the rendering technique based on travelling ultrasonic vibration (Friedman test: $\chi 2=25.14$, $\mathrm{p}=0.0001)$. A further post-hoc analysis with Wilcoxon matched-pairs signed rank test showed that differences were statistically significant for all threshold conditions. (respectively $\mathrm{n}=10, \mathrm{~W}=-42, \mathrm{p}=0.011 ; \mathrm{n}=10, \mathrm{~W}=-39, \mathrm{p}=$ 0.019 and $\mathrm{n}=10, \mathrm{~W}=-45, \mathrm{p}=0.004$ for $0.11 \mathrm{~N}, 0.44 \mathrm{~N}$ and $0.77 \mathrm{~N})$. Thus, rendering with travelling ultrasonic vibration when only one click was allowed decreased the median detection threshold compared to stationary vibration by similar percentage to the one observed when multiple clicks were possible.

We further compared the psychophysical thresholds of the second experiment to those observed in the first one (Fig. 5a) and although they showed a slight increase, the difference was not significant (Mann-Whitney test: $\mathrm{U}=300.5, \mathrm{p}=$ 0.096 for the stationary actuation and $\mathrm{U}=373, \mathrm{p}=0.614$ for the actuation by a travelling wave). Thus, pressing numerous times did not significantly influence the keyclick perception threshold of the users.

As in experiment 1, we measured the peak normal force that the participant exerted and we averaged its value across all participants for each trial number 
a)

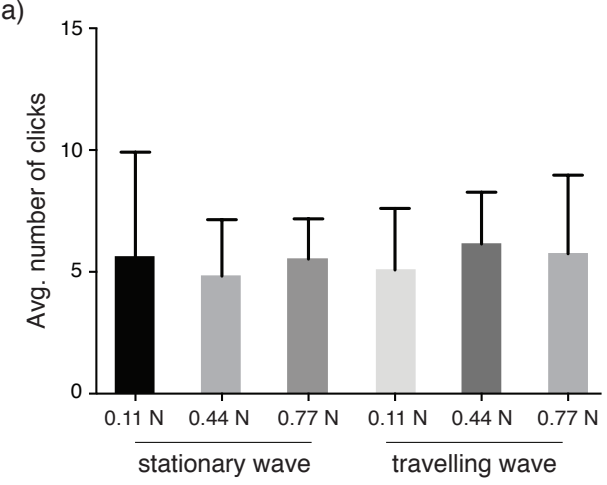

c)

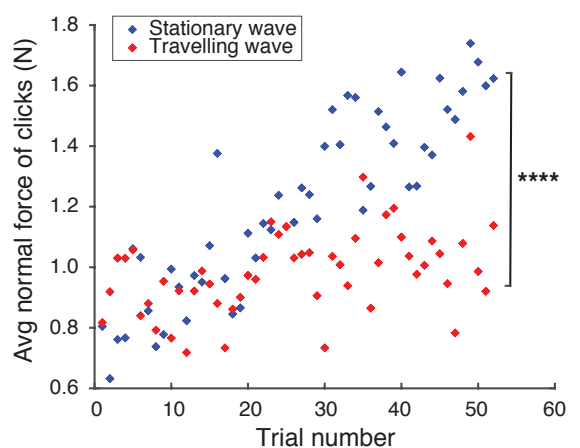

b)

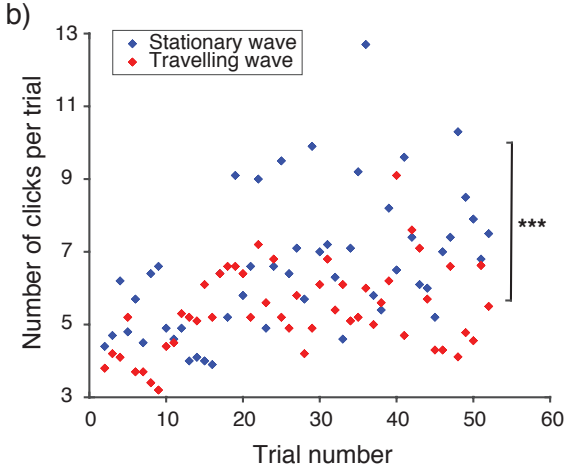

d)

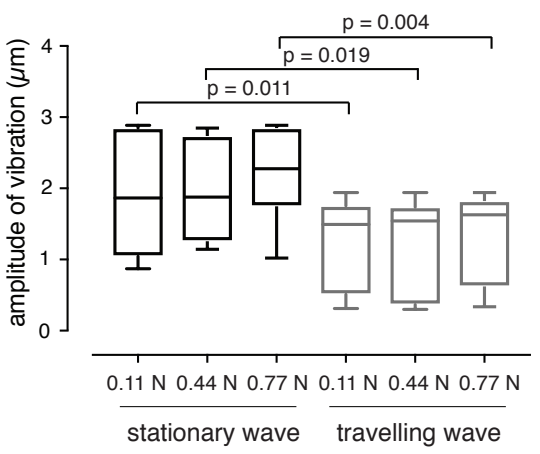

Fig. 4: a) The average number of key presses (clicks) per condition for all six conditions. The boxplots and error bars show respectively the median values and the interquartile ranges. b) Scatter plot of the average number of clicks for a given trial number. c) Scatter plot of the average peak normal force during clicking for a given trial number. d) In experiment 2 (one click), the $50 \%$ perceptual threshold was computed for both actuation techniques. The error bars, the whisker boxes and the horizontal bars respectively show the min. and max. values, their interquartile range and the median value.

of the staircase. In the second experiment, 47 trials were available for all participants and we compared the peak forces between the two actuation techniques (Fig. 5b). The results showed a significantly higher average peak force during pressing for the actuation by a stationary wave (Wilcoxon matched-pairs signed rank statistical test: $\mathrm{N}=43, \mathrm{~W}=946, \mathrm{p}<0.0001)$. In this experiment, even the early trials with a high amplitude of vibration showed a large difference between both techniques.

In both experiments, we asked participants which technique rendered the more realistic keyclick sensation. Seven out of ten participants preferred actuation with the travelling wave in experiment 1 and five out of ten in experiment 2, 
a)

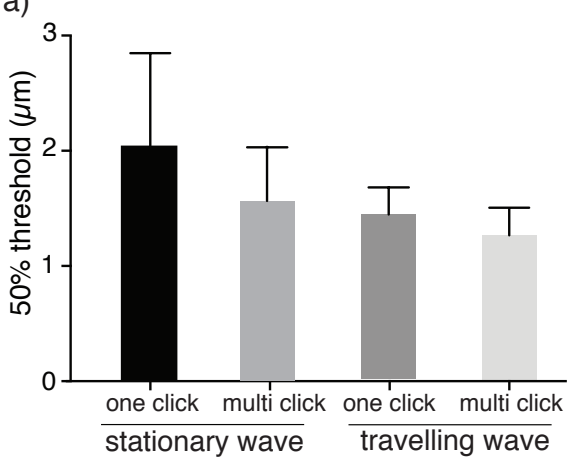

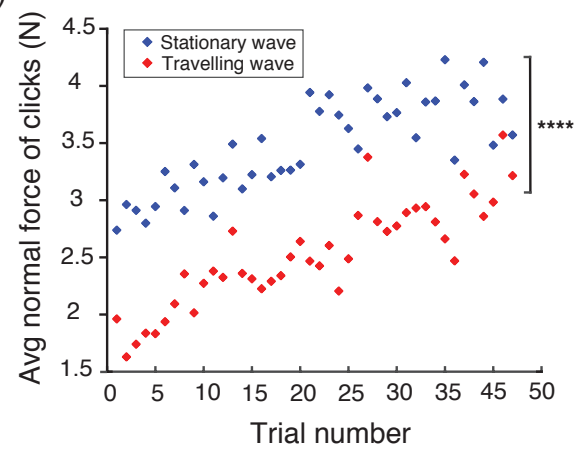

Fig. 5: a) Comparison of the perceptual thresholds between the multiple clicks and one click conditions for both actuation techniques. The boxplots and error bars show respectively the median values and the interquartile ranges. b) Scatter plot of the average peak normal force during pressing for a given trial number.

suggesting that the travelling wave generates a quality of the keyclick sensation similar to the stationary actuation.

\section{Discussion}

Our study shows that the actuation of an ultrasonic keyclick by a travelling wave decreases the amount of vibration amplitude which is necessary to render the click. We also found that the users are less prone to use excessive normal force or click multiple times compared to rendering with a stationary wave. Interestingly, pressing one or multiple times did not significantly influence the psychophysical threshold. This suggests that for a given set of parameters, the keyclick induces a consistent sensation and that the temptation for repeated pressing is mainly done to confirm the sensation of the first click. The difference between both techniques in the pressing normal force was larger when participants were constrained to one click. This suggests that participants felt that they are maximizing their perception of the stationary ultrasonic keyclick by pressing harder hence inducing a larger compression of their fingertip. Although the reflex to push harder when the stimulus becomes subtle was still present, it was less pronounced for the travelling wave, probably because they felt that this type of stimulation mostly induces a lateral force on the fingertip.

We explain these results by the capacity of the travelling wave to produce more tangential force contrast than a stationary wave, whose feedback intensity depends on the lateral component of the fingerpad's compression. This larger contrast stems of the wave's constant pushing force $F_{t+}$ that exists independently of lateral motion of the finger. The strong lateral force component of the actuation by a travelling wave raises the question of the perceived quality of the 
keyclick sensation since the force exerted by a physical keyboard is purely normal. To that end, we compared the subjective perception of keyclick rendering generated by the travelling wave to the one generated by the stationary wave by asking participants to report their preferred rendering. The reports did not show an overwhelming preference for one of the techniques confirming that the travelling wave method generates a genuine keyclick sensation on the fingertip.

\section{Conclusion}

Overall, actuation by travelling ultrasonic wave is a promising technique to create tactile feedback on a finger that is not moving laterally. For the keyclick, this method decreases the threshold for perceiving the click by $25-30 \%$ compared to actuation with a stationary ultrasonic wave and the normal force exerted by the participants is also significantly reduced compared to the stationary condition. In future applications, one can imagine to vary the speed and direction of a travelling wave at a high frequency to create different types of signal on the unmoving finger such as meaningful interactive cues, force-based vibrations or cues for localizing a screen icon. These applications could be complementary to the textures and renderings already available through stationary ultrasonic friction modulation.

Acknowledgment The authors would like to thank the CNRS/IRCICA - USR 3380 research center, which hosted the research. This work was funded by the MATRICE project (ERDF / Région Nord-Pas-de-Calais-Picardie, France).

\section{References}

1. Buxton, W., Hill, R., Rowley, P.: Issues and techniques in touch-sensitive tablet input. ACM SIGGRAPH Computer Graphics 19(3), 215-224 (1985)

2. Chen, H.Y., Park, J., Dai, S., Tan, H.Z.: Design and evaluation of identifiable key-click signals for mobile devices. IEEE Transactions on Haptics 4(4), 229-241 (2011)

3. Ghenna, S., Vezzoli, E., Giraud-Audine, C., Giraud, F., Amberg, M., LemaireSemail, B.: Enhancing variable friction tactile display using an ultrasonic travelling wave. IEEE transactions on haptics 10(2), 296-301 (2017)

4. Gueorguiev, D., Vezzoli, E., Sednaoui, T., Grisoni, L., Lemaire-Semail, B.: Feeling multiple edges: The tactile perception of short ultrasonic square reductions of the finger-surface friction. In: 2017 IEEE World Haptics Conference (WHC). pp. 125129 (2017)

5. Gueorguiev, D., Bochereau, S., Mouraux, A., Hayward, V., Thonnard, J.L.: Touch uses frictional cues to discriminate flat materials. Scientific reports 6 (2016)

6. Gueorguiev, D., Vezzoli, E., Mouraux, A., Lemaire-Semail, B., Thonnard, J.L.: The tactile perception of transient changes in friction. Journal of The Royal Society Interface 14(137), 20170641 (2017)

7. Hoggan, E., Brewster, S.A., Johnston, J.: Investigating the effectiveness of tactile feedback for mobile touchscreens. In: Proceedings of the SIGCHI conference on Human factors in computing systems. pp. 1573-1582. ACM (2008) 
8. Koskinen, E., Kaaresoja, T., Laitinen, P.: Feel-good touch: Finding the most pleasant tactile feedback for a mobile touch screen button. In: Proceedings of the 10th International Conference on Multimodal Interfaces. pp. 297-304. ICMI '08, ACM, New York, NY, USA (2008)

9. Kuchenbecker, K.J., Fiene, J., Niemeyer, G.: Improving contact realism through event-based haptic feedback. IEEE transactions on visualization and computer graphics 12(2), 219-230 (2006)

10. Ma, Z., Edge, D., Findlater, L., Tan, H.Z.: Haptic keyclick feedback improves typing speed and reduces typing errors on a flat keyboard. In: World Haptics Conference (WHC), 2015 IEEE. pp. 220-227. IEEE (2015)

11. Meyer, D.J., Peshkin, M.A., Colgate, J.E.: Tactile paintbrush: A procedural method for generating spatial haptic texture. In: Haptics Symposium (HAPTICS), 2016 IEEE. pp. 259-264. IEEE (2016)

12. Monnoyer, J., Diaz, E., Bourdin, C., Wiertlewski, M.: Ultrasonic friction modulation while pressing induces a tactile feedback. In: International Conference on Human Haptic Sensing and Touch Enabled Computer Applications. pp. 171-179. Springer (2016)

13. Monnoyer, J., Diaz, E., Bourdin, C., Wiertlewski, M.: Optimal skin impedance promotes perception of ultrasonic switches. In: World Haptics Conference (WHC), 2017 IEEE. pp. 130-135. IEEE (2017)

14. Tashiro, K., Shiokawa, Y., Aono, T., Maeno, T.: Realization of button click feeling by use of ultrasonic vibration and force feedback. In: EuroHaptics conference, 2009 and Symposium on Haptic Interfaces for Virtual Environment and Teleoperator Systems. World Haptics 2009. Third Joint. pp. 1-6. IEEE (2009)

15. Vezzoli, E., Sednaoui, T., Amberg, M., Giraud, F., Lemaire-Semail, B.: Texture rendering strategies with a high fidelity-capacitive visual-haptic friction control device. In: International Conference on Human Haptic Sensing and Touch Enabled Computer Applications. pp. 251-260. Springer (2016)

16. Vezzoli, E., Vidrih, Z., Giamundo, V., Lemaire-Semail, B., Giraud, F., Rodic, T., Peric, D., Adams, M.: Friction reduction through ultrasonic vibration part 1: Modelling intermittent contact. IEEE transactions on haptics 10(2), 196-207 (2017)

17. Von Békésy, G., Wever, E.G.: Experiments in hearing, vol. 8. McGraw-Hill New York (1960)

18. Weir, D.W., Peshkin, M., Colgate, J.E., Buttolo, P., Rankin, J., Johnston, M.: The haptic profile: capturing the feel of switches. In: Haptic Interfaces for Virtual Environment and Teleoperator Systems, 2004. HAPTICS'04. Proceedings. 12th International Symposium on. pp. 186-193. IEEE (2004)

19. Wiertlewski, M., Friesen, R.F., Colgate, J.E.: Partial squeeze film levitation modulates fingertip friction. Proceedings of the National Academy of Sciences 113(33), 9210-9215 (2016)

20. Wilson, A.D., Izadi, S., Hilliges, O., Garcia-Mendoza, A., Kirk, D.: Bringing physics to the surface. In: Proceedings of the 21st annual ACM symposium on User interface software and technology. pp. 67-76. ACM (2008) 